\title{
GNSS-to-GNSS time offsets: study on the broadcast of a common reference time
}

\author{
Ilaria Sesia ${ }^{1} \cdot$ Giovanna Signorile $^{1} \cdot$ Tung Thanh Thai $^{1} \mathbb{C} \cdot$ Pascale Defraigne $^{2} \cdot$ Patrizia Tavella $^{3}$
}

Received: 25 May 2020 / Accepted: 27 December 2020 / Published online: 17 February 2021

(c) The Author(s) 2021

\begin{abstract}
We present two different approaches to broadcasting information to retrieve the GNSS-to-GNSS time offsets needed by users of multi-GNSS signals. Both approaches rely on the broadcast of a single time offset of each GNSS time versus one common time scale instead of broadcasting the time offsets between each of the constellation pairs. The first common time scale is the average of the GNSS time scales, and the second time scale is the prediction of UTC already broadcast by the different systems. We show that the average GNSS time scale allows the estimation of the GNSS-to-GNSS time offset at the user level with the very low uncertainty of a few nanoseconds when the receivers at both the provider and user levels are fully calibrated. The use of broadcast UTC prediction as a common time scale has a slightly larger uncertainty, which depends on the broadcast UTC prediction quality, which could be improved in the future. This study focuses on the evaluation of two different common time scales, not considering the impact of receiver calibration, at the user and provider levels, which can nevertheless have an important impact on GNSS-to-GNSS time offset estimation.
\end{abstract}

Keywords GNSS · GGTO · Timing · Time scale $\cdot$ Interoperability

\section{Introduction}

Positioning and timing performances at the user level can be significantly increased by using all Global Navigation Satellite System (GNSS) satellites in view and not only those satellites belonging to a single constellation. This approach, however, requires some interoperability between the different systems, notably knowledge of the offsets between different GNSS time scales. In what follows, the four currently existing global navigation systems will be considered with their associated time scale: BeiDou with BeiDou Time (BDT), Galileo with Galileo System Time (GST), GLONASS with GLONASS Time (GLONASST), and Global Positioning System (GPS) with GPS Time (GPST).

We generically call XYTO the time offset between the reference time of system $\mathrm{X}$ with respect to the reference

Ilaria Sesia

i.sesia@inrim.it

Istituto Nazionale di Ricerca Metrologica, Turin, Italy

Royal Observatory of Belgium, ORB, Brussels, Belgium

3 Bureau International des Poids et Mesures (BIPM), Pavillon de Breteuil, 92312 Sèvres Cedex, France time of system Y. The users can obtain the information on the needed XYTO from the GNSS, which may transmit this information in the navigation message. Alternately, the users can estimate such time offsets themselves, adding additional unknowns to the position, velocity and time (PVT) set of equations if the number of observed satellites is sufficient.

Galileo is currently disseminating the GPS to Galileo Time Offset (GGTO) through its navigation message, enabling users equipped with a combined GPS/Galileo receiver to achieve a PVT solution using measurements from both systems at the same time (Píriz et al. 2008; GSA 2018). Other GNSSs are available, so the time offsets between all the different systems should be made available to the users in the multi-GNSS situation, e.g., GPS to GLONASS and GPS to BeiDou.

Broadcasting the time offsets with respect to every other system would impose a high level of complexity and require a significant overload on each GNSS provider with many additional parameters to be estimated and transmitted in the navigation messages. However, the users cannot estimate the time offset XYTOs unless they have enough usable satellites to solve the enlarged equation set. This availability of usable satellites can be a limitation in the urban environment, precisely where the benefit of a multiconstellation is 
at a maximum. The advantages and disadvantages for the timing and positioning of a transmitted XYTO with respect to a solution at the user level are a matter of debate and experimentation; see, for example, Gioia and Borio (2016).

Considering such constraints, at the 2017 annual meeting of the International Committee on Global Navigation Satellite Systems (ICG), it was proposed that each GNSS system broadcasts only the time offset between its own system time scale and a common reference time scale. The common reference was, however, not identified, as different options are available. Here, we consider two possible options for this common reference. The first is a simple average of the

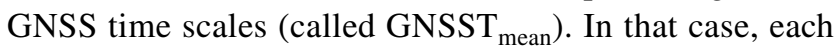
GNSS would be estimating and broadcasting the time offset between its time scale and GNSST mean $_{\text {. The second option }}$ is to use the prediction of the Coordinated Universal Time (UTC), which is already broadcast by all GNSSs in their own navigation messages, therefore not requiring any change for the GNSS providers. Regarding our second approach, other studies are also available (Kosheliaevskii 2019; Tavella and Petit 2019; Yuan et al. 2019 at ICG 2019 annual meeting) on the use of the prediction of UTC included in the GNSS navigation message.

The basic idea behind these two approaches was already discussed at the 32nd European Time and Frequency Forum, paper Signorile et al. (2018). Some estimations were reported in a simplified scheme by using the same multiGNSS receiver to determine GNSST ${ }_{\text {mean }}$ and to simulate the user estimates. The present article instead considers a more complete and realistic approach for the use of GNSST mean $_{\text {, }}$ where each GNSS determines the difference between its system time scale and GNSST mean $_{\text {independently by using }}$ data from its own multi-GNSS station. To simulate this scenario, we use different receivers distributed worldwide to act as the stations used by the different GNSS providers, and additional stations are then used to play the role of user receivers. For the two proposed approaches, we compare the results with respect to the XYTO estimated by the user as an additional unknown and give an evaluation of the uncertainty sources that play a role in both schemes.

As we want to focus our study on the two different common reference time scales, we assume that all the GNSS receivers that are involved are fully calibrated. This assumption is not trivial, but it is fundamental, as calibration residuals are the major source of uncertainty for interoperability. To set up the experimental scenario, we used, whenever possible, receivers that were already calibrated. In other cases, we numerically determined the hardware delays of the receivers in use; the procedure we applied is detailed in appendix.

We first introduce the two proposed candidates for the reference time scale common to all GNSSs. Then, we present and discuss the results obtained and the performances that can be achieved with each of the two approaches. The conclusions are stated in the final section.

\section{Common reference: GNSST $_{\text {mean }}$}

The first method relies on the assumption that each GNSS broadcasts a prediction of the time offset between its own reference time scale (GNSST) and the average of the four GNSS reference time scales, namely, GNSST mean $_{\text {. Only the }}$ four global constellations (BeiDou, Galileo, GLONASS, GPS) are considered for the average time scale. Regional systems could use the same average of four global constellations as a reference and broadcast the time offset between their reference time and the 4-system average GNSST mean $_{\text {. }}$.

We consider a simple scenario as depicted in Fig. 1, where the provider of each system (i) can autonomously determine the time offset $\mathrm{GNSST}_{i}-\mathrm{GNSST}_{\text {mean }}$ using a calibrated multi-GNSS receiver connected to a pivot clock measuring

Pivot clock - GNSST

where $\mathrm{GNSST}_{i}$ is the reference time scale of $\mathrm{GNSS}_{\mathrm{i}}$, as obtained through Signal in Space (SIS), $i=1 \ldots 4$ for the four different GNSSs. Pivot clock is any reference clock or time scale feeding the used multi-GNSS receiver. The pivot clock could also be a physical representation of $\mathrm{GNSST}_{i}$, but

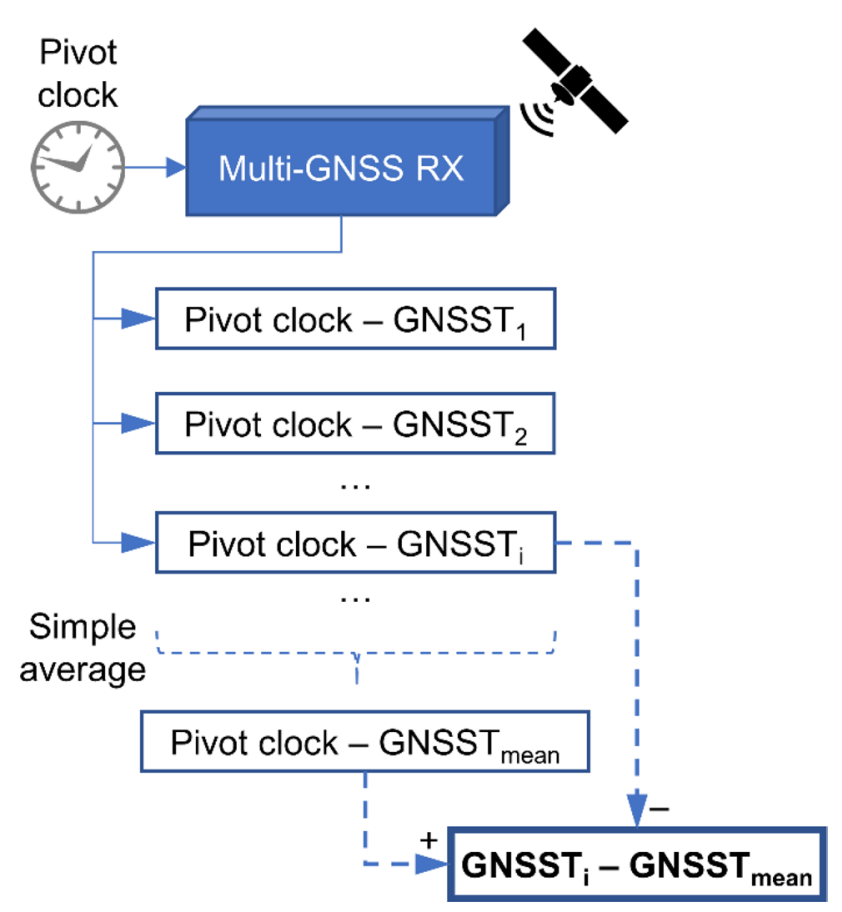

Fig. 1 High-level scheme for GNSST $_{i}-$ GNSST $_{\text {mean }}$ computation as a simple average of each GNSST $_{i}$ component 
for generality, we assume that $\mathrm{GNSST}_{i}$ is obtained from the navigation solution of the receiver.

GNSST $_{\text {mean }}$ is then obtained as a combination of the four GNSS-collected measurements (1):

Pivot clock - GNSST $_{\text {mean }}=\frac{1}{4} \sum_{i=1}^{4}\left(\right.$ Pivot clock - GNSST $\left._{i}\right)$

where a simple averaging operation is proposed to limit the complexity and, moreover, to reduce possible discrepancies in the results obtained from different implementations adopted by the different system providers.

At this point, the offset $\mathrm{GNSST}_{i}-\mathrm{GNSST}_{\text {mean }}$ to be broadcast can be determined as the difference between Eqs. (1) and (2):
Force 2013), Galileo is instead predicting the offset between GST and UTC, using five European UTC $(k)$ time scales as intermediate references (GSA 2016a), GLONASS is broadcasting a prediction of GLONASST versus UTC(SU) (GLONASS Coordination Scientific Information Center 1998), and BeiDou is broadcasting a prediction of UTC based on UTC (NTSC) (Chinese Satellite Navigation Office 2018). Here, USNO is the US Naval Observatory, SU stands for the Russian time scale realized by the VNIIFTRI Institute, and NTSC is the Chinese National Timing Service Center. Conversely, Galileo uses as an intermediate reference the German, French, Swedish, Spanish, and Italian realizations of UTC $(k)$. The UTC references chosen by the four GNSSs are different, but as they are all based on real-time local realizations of UTC, aiming to be good approximations of

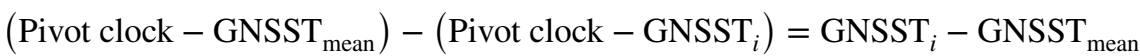

where the behavior of pivot clock has no direct effect on the quantities of interest. Therefore, there are no particular constraints on the pivot clock to be used. The pivot clock can be chosen by each provider according to its own system architecture and operative needs.

Once computed, the quantity $\mathrm{GNSST}_{i}-\mathrm{GNSST}_{\text {mean }}$ must then be predicted for the future period and broadcast in the navigation message by each GNSS provider. Then, at the user level, the difference between two system time scales $X$ and $Y(X Y T O)$ can easily be estimated as
UTC, their difference is usually small (Panfilo and Arias 2019). In addition, each GNSS has a different prediction and update policy for the broadcast value of Brdc_UTC $\mathrm{UNNS}_{\mathrm{GNS}}$ - GNSST. Nevertheless, as we will see in the next sections, such contributions have, in general, a limited impact on the effectiveness of the method.

The user can then estimate XYTO by combining the different Brdc_UTC $\mathrm{GNSS}_{\mathrm{GNS}}-$ GNST predictions broadcast by each GNSS as follows, considering the broadcast predictions of UTC as equivalent:

$X Y T O=\operatorname{GNSST}_{X}-\operatorname{GNSST}_{Y}=\left[\mathrm{GNSST}_{X}-\mathrm{GNSST}_{\text {mean }}\right]_{\mathrm{brdc}}-\left[\mathrm{GNSST}_{Y}-\mathrm{GNSST}_{\text {mean }}\right]_{\mathrm{brdc}}$

where the suffix "brdc" means as obtained by the user directly from the GNSS broadcast navigation message.

\section{Common reference: coordinated Universal Time (UTC) as broadcast by GNSS}

While the first approach, based on GNSST ${ }_{\text {mean }}$, implies the definition of a new common time scale, the second approach proposes as a common reference time scale an already existing one: the prediction of UTC provided in the navigation message of each GNSS. We call this quantity Brdc_UTC GNSS. Our second method proposes the use of this time scale as a common reference to determine the intersystem time offsets.

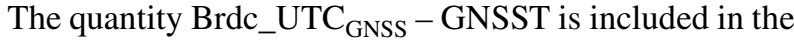
navigation message and is obtained by the different GNSSs in different ways based on different algorithms and on different UTC realizations. GPS is broadcasting the difference of GPST with respect to UTC(USNO) (United States Space

$$
\begin{aligned}
\text { XYTO }= & \left(\text { Brdc } \_U T C_{\mathrm{GNSS}_{Y}}-\mathrm{GNSST}_{Y}\right) \\
& -\left(\mathrm{Brdc}_{-} \mathrm{UTC}_{\mathrm{GNSS}_{X}}-\mathrm{GNSST}_{X}\right)
\end{aligned}
$$

The main advantage of this method is that all the information needed is already available in the navigation message, so no changes are required at the GNSS system level based on the assumption that the broadcasted predictions of UTC could be considered by the users as equivalent within the declared level of uncertainties. This assumption also applies to other regional satellite navigation systems that provide predictions of their reference time scales versus UTC.

\section{Experimental results}

Two tests have been designed to demonstrate the feasibility and achievable performances through the two approaches described previously. Approximately 40 days of real multiGNSS calibrated data were analyzed (January 23-February $28,2018)$. The first test, based on the GNSST mean method, 
uses CGGTTS v2E (Defraigne and Petit 2015; Verhasselt and Defraigne 2019) files of the multi-GNSS stations located worldwide. Thus, only the clock solution is determined, while the station positions are considered fixed. The coordinates were determined using GPS data and precise point positioning with Atomium software (Defraigne et al. 2008). For the second test, based on the Brdc_UTC ${ }_{\text {GNSS }}$ method, the GNSST - UTC predictions were collected from the multi-GNSS RINEX Navigation Files provided by the International GNSS Service (IGS). The results are presented and discussed in the following sections.

\section{First test: using the GNSST $_{\text {mean }}$ method}

Four multi-GNSS stations were chosen as representatives of the GNSS providers (BeiDou, Galileo, GLONASS, and GPS). These stations are listed in Table 1, and their locations are visible in Fig. 2, which, to be more realistic, are in the same regions as their respective GNSS providers. These stations are used for computing the common reference

Table 1 List of multi-GNSS stations used to test the method based on GNSST $_{\text {mean }}$

\begin{tabular}{llll}
\hline Station & Location & Role in this test \\
\hline NTP3 & China & BeiDou provider \\
BRUX & Belgium & Galileo provider \\
BOR1 & Poland & GLONASS provider \\
STJ3 & Canada & GPS provider \\
GMSD & Japan & User \\
\hline
\end{tabular}

The color of each station matches the colors of Fig. 2, indicating their locations on the map

Fig. 2 Geographical maps of the multi-GNSS stations used to test the method based on GNSST $_{\text {mean }}$
GNSST $_{\text {mean }}$ to be broadcast by each constellation, according to the procedure described previously. The advantage of using geographically distributed stations is that they observe different satellites at the same time, which reflects the true situation of geographically distributed GNSS providers. An additional station, located in Japan, is acting as the user and is used to estimate $X Y T O$ from the combination of [GNSST $i$ - GNSST mean $_{\text {brdc }}$ as in Equation (4) and to compare it with the XYTO determined at the user level from the receiver pseudorange measurements as an additional unknown.

The stations were chosen from the IGS network considering some key prerequisites such as having a stable external reference clock and being capable of receiving signals from the four GNSS constellations. Their RINEX observation and navigation files were retrieved from the IGS CDDIS data center ftp://cddis.nasa.gov/gnss/data/daily (Noll 2010); the data from the Chinese station NTP3 were kindly provided by the National Time Service Center (NTSC).

Since we are dealing with time offset measurements, accurate calibration of the receivers is needed to avoid biases in the final XYTO estimates. Among the chosen stations, only BRUX, whose pivot clock is the Belgian time scale UTC(ORB), is currently calibrated and only for GPS and Galileo signals. BRUX was further calibrated for GLONASS by using the information published by the International Bureau of Weights and Measures (BIPM), namely, UTC - UTC(ORB) (Section 1 of Circular T) and UTC - GLONASST, combined with the measurement UTC(ORB) - GLONASST from the BRUX receiver. The BeiDou signals were also calibrated using the information on BDT - UTC provided by Cai et al. at the ICG 2017 annual meeting, combined with the UTC - UTC(ORB) data found in Circular T. Then, a differential calibration with respect to BRUX was carried out for the other
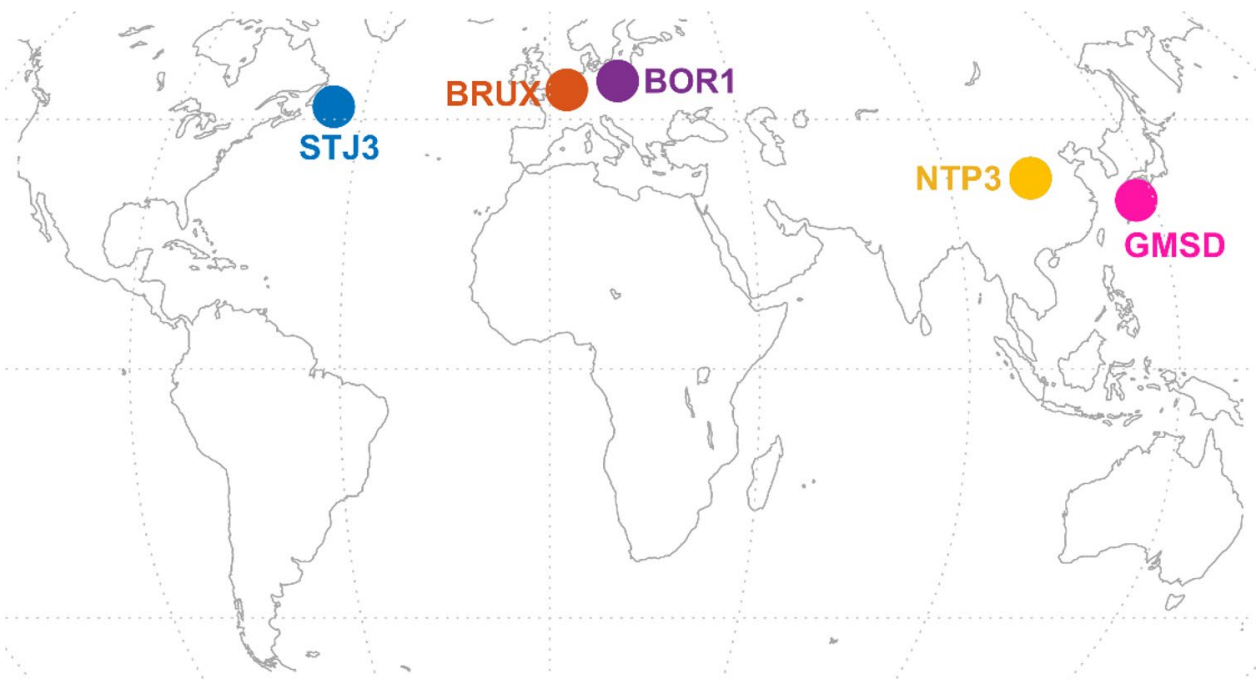
stations involved in the case study. Details about the calibration approach are reported in Appendix.

The first step of our test case consists of carrying out the computation and prediction of $\mathrm{GNSST}_{\mathrm{i}}-\mathrm{GNSST}_{\text {mean }}$ as would be carried out by each provider as described through Eqs. (1) and (2). The measures pivot clock - GNSST is obtained from the CGGTTS files. For example, in the case of Galileo, GST - GNSST mean $_{\text {is obtained }}$ using UTC(ORB) as the pivot clock and similarly for the other stations playing the role of GNSS provider, as mentioned in Table 1. GNSST $i$ - GNSST mean then needs to be predicted for the subsequent day and broadcast in the navigation message so that the user can combine the information obtained by the different GNSSs and then estimate XYTO. For simplicity, we propose to use, as a prediction for the subsequent day, a constant value equal to the average of the previous day measures to be updated on a daily basis. Based on the data of calibrated receivers acting as GNSS providers in our case study (as per Table 1), the results of the $\mathrm{GNSST}_{i}-\mathrm{GNSST}_{\text {mean }}$ offset estimation and predictions are depicted in Fig. 3. The continuous pale lines report the quantity $\mathrm{GNSST}_{i}-\mathrm{GNSST}_{\text {mean }}$ for the four GNSS simulated providers obtained according to Eqs. (1), (2) and (3). From these measures, a daily average is computed and reported as prediction, by the solid line, for the subsequent day. The prediction is updated daily based on the average value of the previous day.

We imagine that a user (GMSD in this test) can receive these predicted values of $\mathrm{GNSST}_{i}-\mathrm{GNSST}_{\text {mean }}$ from the navigation messages and can estimate XYTO. The user willing to combine satellites from systems $X$ and $Y$ needs to apply Equation (4) to estimate XYTO $=\mathrm{GNSST}_{X}-\mathrm{GNSST}_{Y}$ from the predicted values of $\mathrm{GNSST}_{i}-\mathrm{GNSST}_{\text {mean }}$ broadcast by providers $X$ and $Y$.
Under good conditions of visibility, the same XYTO quantity can also be estimated through the user receiver by using Signal in Space (SIS) observables by adding an additional unknown to the determination of position and time. We call this independent estimation at the user level XYTO SIS.

The results for the GYTO case defined as the offset of GST versus the other GNSS time scales are reported in Fig. 4. The results obtained between the other constellations, not shown here, lead to exactly the same conclusion. The GYTO estimated from the broadcast value GNSST

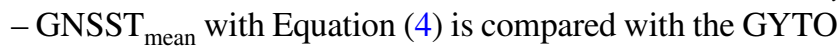
SIS obtained at the user level from GNSS measurements. In the latter case, the user is considered in its most accurate situation, i.e., a fixed user with good visibility and a fully calibrated receiver. Even if in this most accurate situation, there would be no need for broadcast GYTO as it could be easily determined by the user, it is considered a good test of the validity of the broadcast information. The user GYTO SIS is computed using the differences of solutions (1) obtained from the CGGTTS results corresponding to the different constellations. This solution is called SIS in Fig. 4, as determined from Signal in Space. Figure 4 also shows the GYTO determined by the user using the broadcast information. The difference between the user SIS accurate estimation and the value determined from the broadcast GNSST $_{\text {mean }}$ is presented in Fig. 5, with the associated average and standard deviations. These differences are due mainly to the noise of the observations and the 1-day stability of the GNSS time scale. Indeed, the more stable the GNSS time scales are, the more accurate the prediction of $\mathrm{GNSST}_{i}-\mathrm{GNSST}_{\text {mean }}$ for the subsequent day.
Fig. 3 Estimations and associated predictions of GNSST - GNSST $_{\text {mean }}$ by the simulated system providers

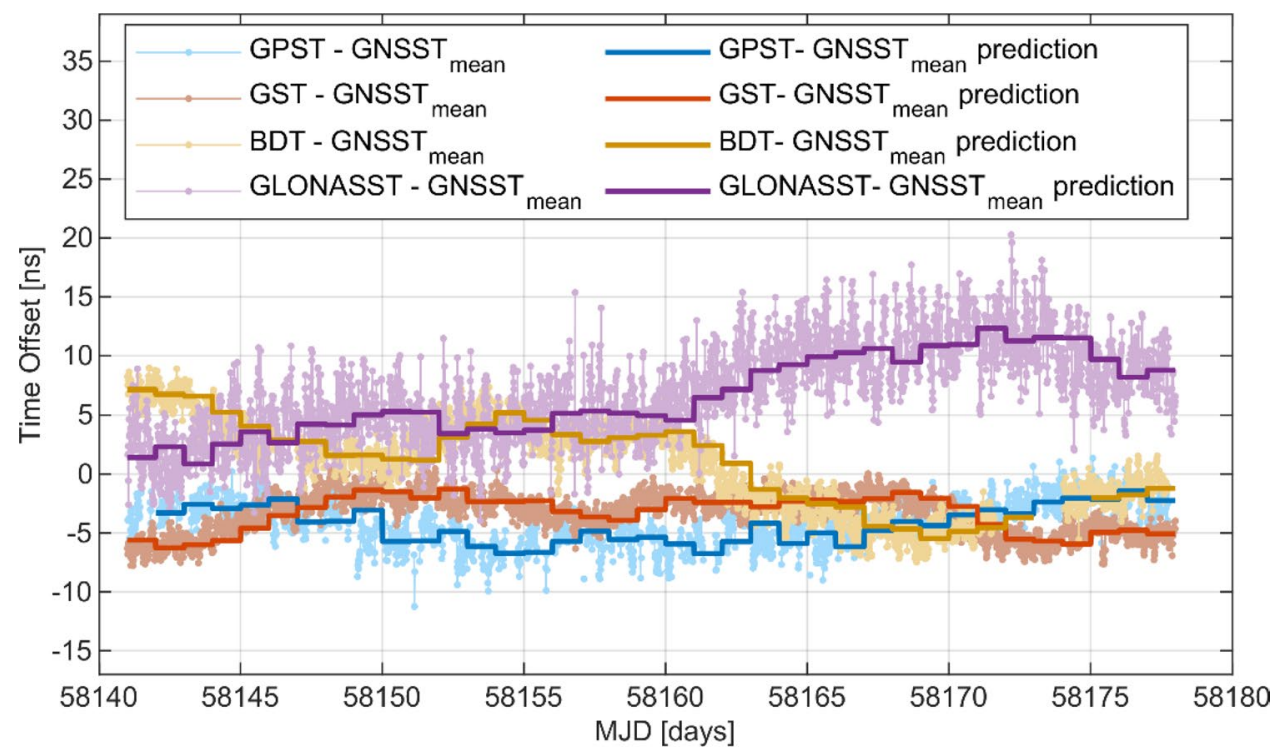


Fig. 4 Comparison between GYTO according to GNSST mean $_{\text {}}$ via providers and GYTO SIS obtained at the user level. The gaps in the SIS solutions are due to some missing RINEX files from one station at these epochs

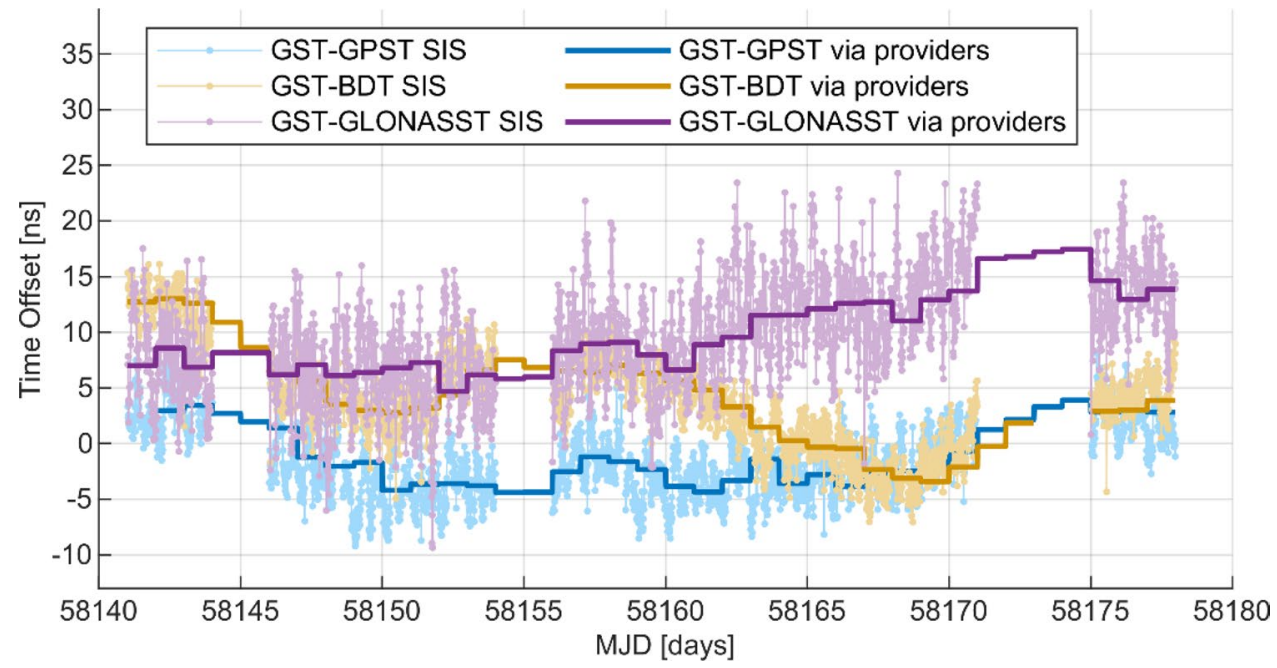

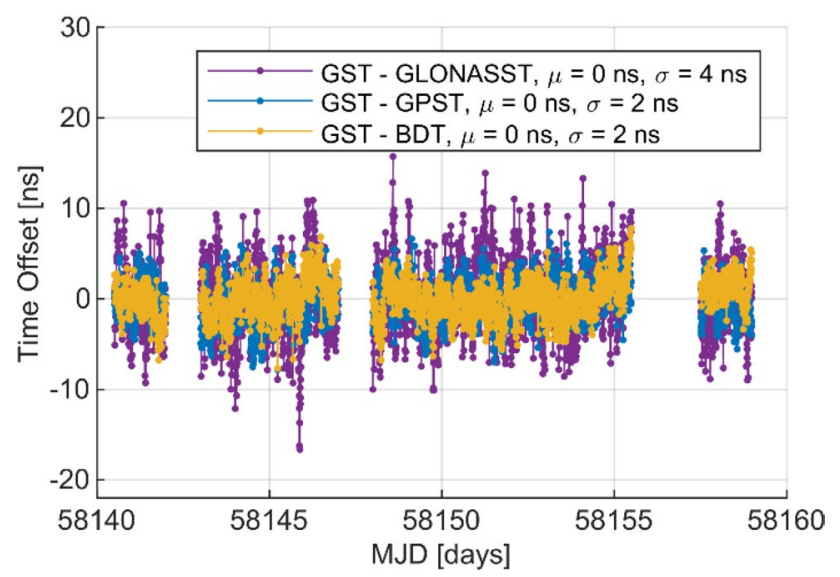

Fig. 5 GYTO differences at the user level between the user SIS solution and brdc solution via the provider based on GNSST mean. The mean value $\mu$ and the standard deviation $\sigma$ of these residuals are also reported

The results based on broadcast information are observed to be highly compatible with the stand-alone user solution; the differences between both are within $5 \mathrm{~ns}(1 \sigma)$ depending on the system due to the different noise in the GNSS measurements of the different constellations. No bias is observed between the two GYTO estimates. We can, therefore, conclude that having the GNSST ${ }_{\text {mean }}$ computed in different locations around the world by the different system providers does not impact the GYTO determination at the user level and provides the user with a very accurate GYTO value. However, these results are based on a set of fully calibrated receivers, both at the system provider level and at the user level.

To simulate a more realistic case, we should also consider possible calibration errors of a few nanoseconds on the provider measurements. These calibration errors would directly translate into biases in the XYTO determined from the information from the system providers. As an example, a bias of $3 \mathrm{~ns}$ in the broadcast $\mathrm{GNSST}_{X}-\mathrm{GNSST}_{\text {mean }}$ and a bias of $-2 \mathrm{~ns}$ in the broadcast $\mathrm{GNSST}_{Y}-\mathrm{GNSST}_{\text {mean }}$ would directly transfer into a bias of $5 \mathrm{~ns}$ for the user determining the XYTO from the broadcast parameters. Considering the calibration possibilities to date, with both absolute (Waller et al. 2019) and relative (Petit et al. 2006) calibrations, we can expect that biases of a maximum of $5 \mathrm{~ns}$ would be present in the estimate of GNSST $_{i}$ - GNSST $_{\text {mean }}$, giving final biases of a maximum of $10 \mathrm{~ns}$ at the user level.

\section{Second test: using the Brdc_UTC ${ }_{\text {GNSS }}$ method}

As mentioned previously, the second approach considers that the predictions of UTC as provided by the different GNSS providers in their navigation messages are equivalent. This assumption is, of course, simplifying, as all these predictions of UTC are fully independent and hence different. The broadcast predictions of UTC are generally in agreement within tens of nanoseconds and, in any case, within the target accuracy of the UTC time dissemination service declared by the providers. For example, according to the Galileo Open Service report (GSA 2018), the uncertainty of the Galileo UTC time dissemination service over the analysis period (January-February 2018) is less than $10 \mathrm{~ns}$, which is well below the specification of $30 \mathrm{~ns}$ for "Galileo SIS UTC Time Dissemination Accuracy”, as defined in (GSA 2016b).

Figure 6 shows, for example, the behavior of UTC(USNO) and UTC(SU) with respect to UTC from BIPM Circular T. These two time scales are disseminated as UTC predictions by GPS and GLONASS providers, respectively. The figure also reports the behavior of UTC(NTSC) used as a link to UTC for BeiDou (Chinese Satellite Navigation Office 2018) and the five European 
$\mathrm{UTC}(k)$ time scales used as intermediate references by Galileo to disseminate GST-UTC prediction. While our approach assumes that the broadcast predictions of UTC are equivalent and could properly cancel out in Equation (5), we see in Fig. 6 that differences of a few nanoseconds can exist between them and can infer some uncertainties to the XYTO obtained using Brdc_UTC ${ }_{\text {GNSS }}$ as the reference. These differences might also be larger in other observation periods.

Before looking at the intersystem biases determined from the broadcast UTC information, let us assess the broadcast values themselves. For each constellation, we can compare the broadcast UTC prediction with the true UTC using a station connected to a UTC $(k)$ time scale as a pivot. This information (UTC - Brdc_UTC $\mathrm{GNSS}_{\mathrm{G} S}$ ) is available in Sect. 4 of
BIPM Circular T for GPS and GLONASS only. In the current study, we combine the solution UTC(ORB) - GNSST $_{i}$ obtained from SIS measurements collected by station BRUX and UTC - UTC(ORB) from Section 1 of BIPM Circular T so that we obtain UTC - GNSST $i$ at the user level. Figure 7 displays this quantity alongside the predicted UTC - GNSST $_{i}$ broadcast in the navigation message. Finally, Fig. 8 shows the difference between these two quantities, i.e., UTC - Brdc_UTC $_{\text {GNss. }}$. The differences between the Brdc_UTC ${ }_{\mathrm{GNSS}}$ of the four global constellations are generally limited to a few nanoseconds, while they are slightly larger for BeiDou (up to $11 \mathrm{~ns}$ ) at the beginning of the period analyzed. These differences can be explained by the different UTC realizations used for the generation of the broadcast messages and the algorithm used for its realization
Fig. 6 UTC-UTC(k) reported in Circular T by BIPM from January 22 to February 26, 2018
Fig. $7 \mathrm{UTC}-\mathrm{GNSST}_{i}$ at the user level and broadcast values Brdc_UTC $\mathrm{GNSS}_{-}-\mathrm{GNSST}_{i}$ from January 22 to February 26, 2018
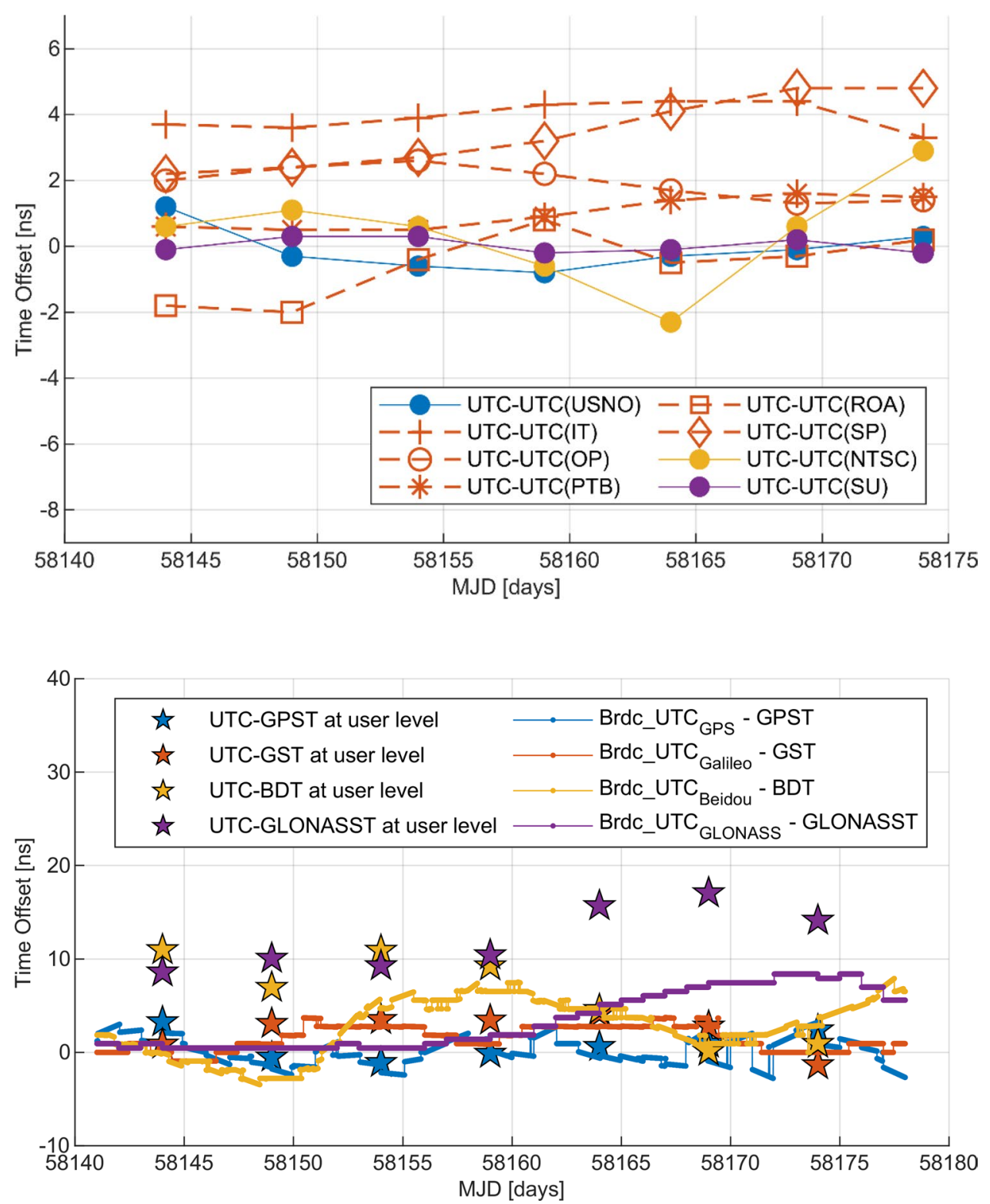
Fig. 8 UTC - Brdc_UTC ${ }_{\text {GNSS }}$, computed via UTC(ORB) from January 22 to February 26, 2018

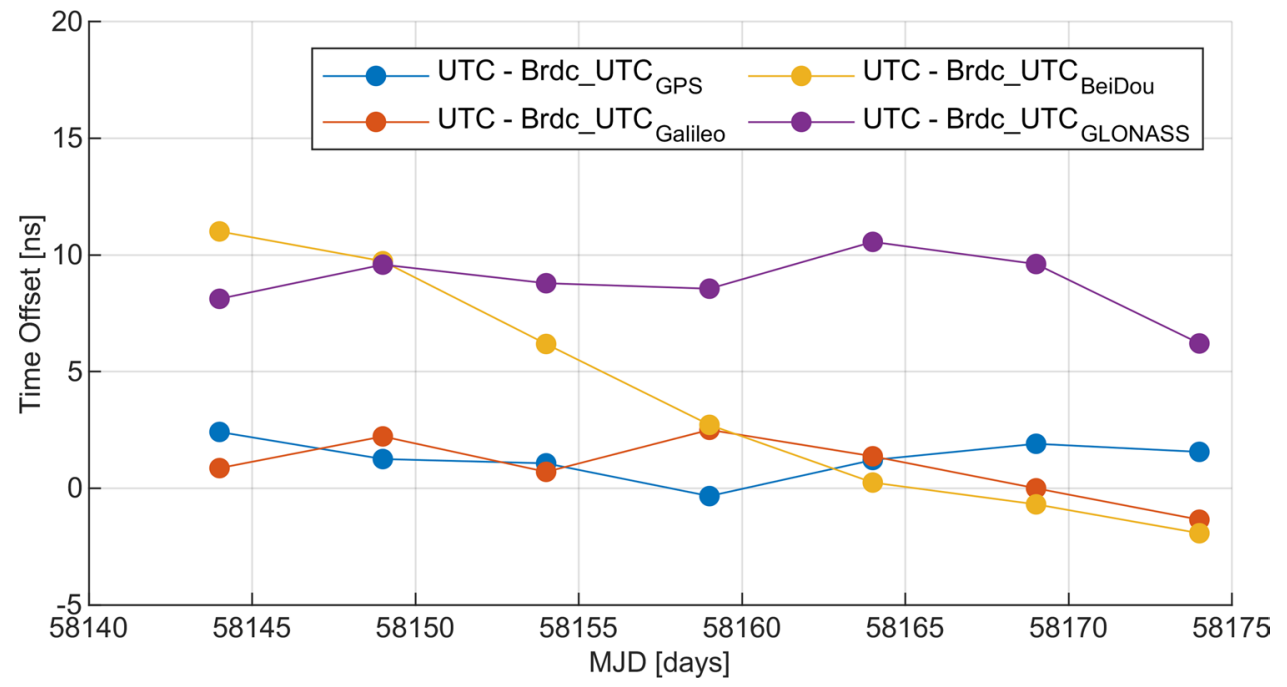

and prediction; possible calibration errors of the receivers involved in the UTC dissemination chains could explain a bias but not the large variations observed. We can, however, expect that in the future, an improvement will be seen at the level of UTC prediction, which will reduce these differences to only a few nanoseconds.

To obtain a first quantification of the uncertainties on the XYTO determined when using the Brdc_UTC ${ }_{\text {GNSS }}$ as the reference, we computed the time offsets GST - GPST, GST - BDT and GST - GLONASST according to Equation (5) and compared it with the accurate estimation of the corresponding GYTO SIS at the user level for station GMSD, as presented in the previous section.

The results are presented in Figs. 9, 10 and 11. The results

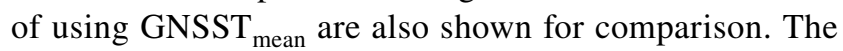
differences between GYTO obtained from Brdc_UTC ${ }_{\mathrm{GNSS}}$ and the accurate SIS estimation are presented for the same constellation pairs in Fig. 12. These differences are up to 10 ns for the period considered, resulting from the differences in UTC predicted and broadcast by each system and calibration residuals of timing equipment used in the determination of such quantity. If we do not consider the biases, we could estimate the statistical uncertainty ( $\sigma$ in the legend of Fig. 12) again at the level of a few nanoseconds, similar to the statistical uncertainty of the first method. We see that in this case, the calibration residuals play an important role.

Since the results of GYTO using Brdc_UTC ${ }_{\text {GNSS }}$ as a reference depend on the capability of each GNSS to predict and disseminate UTC and on the status of the calibration of the receivers used in the UTC dissemination chains, we selected a more recent period (4 months from January to April 2019) for further analysis. We applied the same procedure described before for the method based

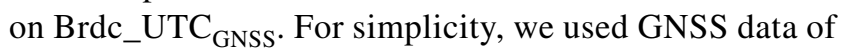
BRUX connected to UTC(ORB) acting as a user in this case. Calibration of the user multi-GNSS BRUX receiver

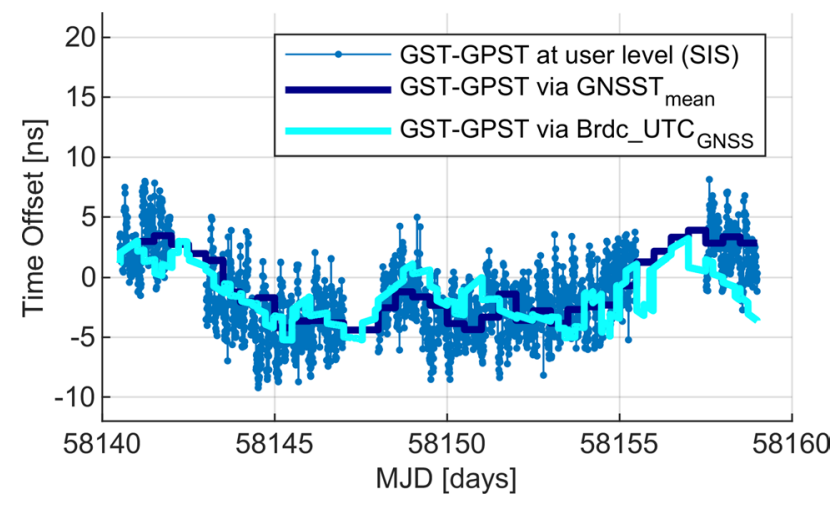

Fig. 9 Comparison of GST - GPST as computed by the different approaches from January 22 to March 2, 2018

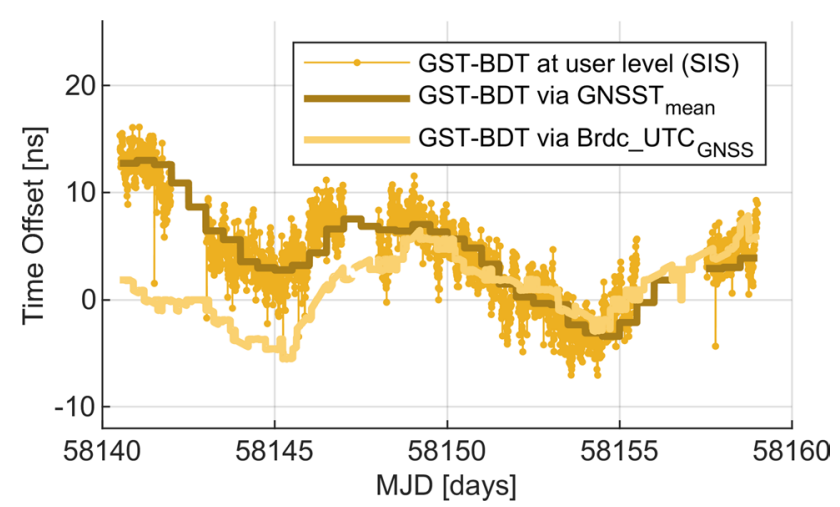

Fig. 10 Comparison of GST - BDT as computed by the different approaches from January 22 to March 2, 2018

was achieved by comparing it to a BIPM multi-GNSS receiver absolutely calibrated and using a GPS-calibrated link between the BIPM and BRUX receivers. 


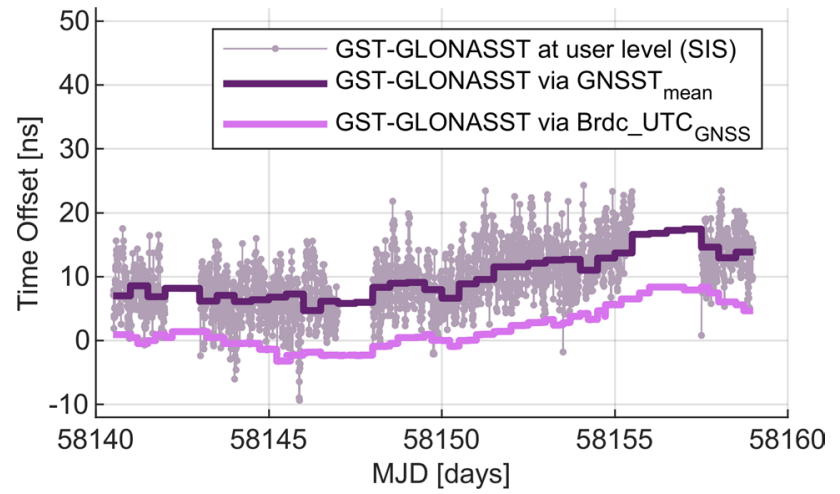

Fig. 11 Comparison of GST - GLONASST as computed by the different approaches from January 22 to March 2, 2018

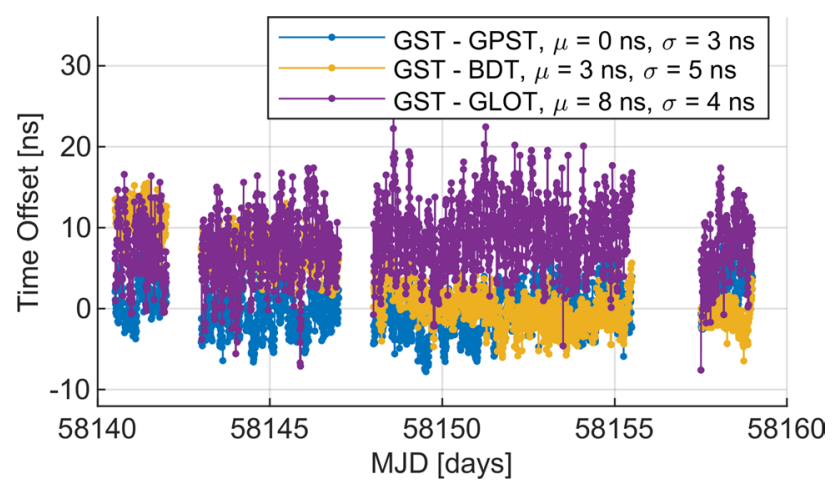

Fig. 12 GYTO differences at the user level between the user SIS

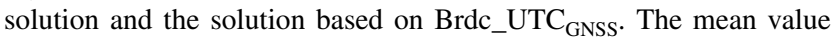
and standard deviation of these differences are also reported in the legend

Figure 13 reports the broadcast values Brdc_UTC GNSS $-\mathrm{GNSST}_{i}$ together with UTC $-\mathrm{GNSST}_{i}$ at the user level, and Fig. 14 shows the difference between the two quantities, which represents UTC - Brdc_UTC $_{G N S S}$. With respect to the previously analyzed period (Fig. 8), some differences between the broadcast value and UTC are still present and vary with time.

Repeating the analysis for GYTO, we compare in Figs. 15, 16 and 17 the GST - GPST, GST - BDT and GST - GLONASST obtained at the user level (using SIS measurements of BRUX) and via Brdc_UTC ${ }_{\mathrm{GNSS}}$ using Equation (5). The difference between these two approaches for each GYTO pair is shown in Fig. 18. In general, both periods, i.e., early 2018 and early 2019, show consistent results. The observed offsets for each GNSS change, as they follow the different UTC realizations used as a reference and possible calibration and prediction errors at each system level. From statistical fluctuations, we can estimate the uncertainty of XYTO at a few nanoseconds, as before and as in the first method. We have to stress that the use of

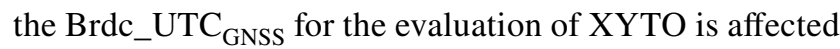
to a larger extent by residual calibration errors. Not only the users and provider receivers but also all the chains connecting each GNSS provider to a UTC realization are to be calibrated, allowing the prediction of Brdc_ $\mathrm{UTC}_{\mathrm{GNSS}}$ - GNSST $_{i}$. As we have said, we expect that UTC dissemination estimation and prediction will improve in the future for each GNSS system.

\section{Comparison of test results}

To compare the results obtained from the GNSST $_{\text {mean }}$ method and those from the Brdc_UTC ${ }_{\text {GNSS }}$ method, Table 2 shows the differences between the SIS solutions and solutions based on the broadcast information of each method for the period analyzed. Assuming that all the receivers are fully calibrated in our first test, no offset is found between GYTO
Fig. $13 \mathrm{UTC}-\mathrm{GNSST}_{i}$ at the user level and broadcast values Brdc_UTC $\mathrm{GNSS}_{-}-\mathrm{GNSST}_{i}$ in January-April 2019

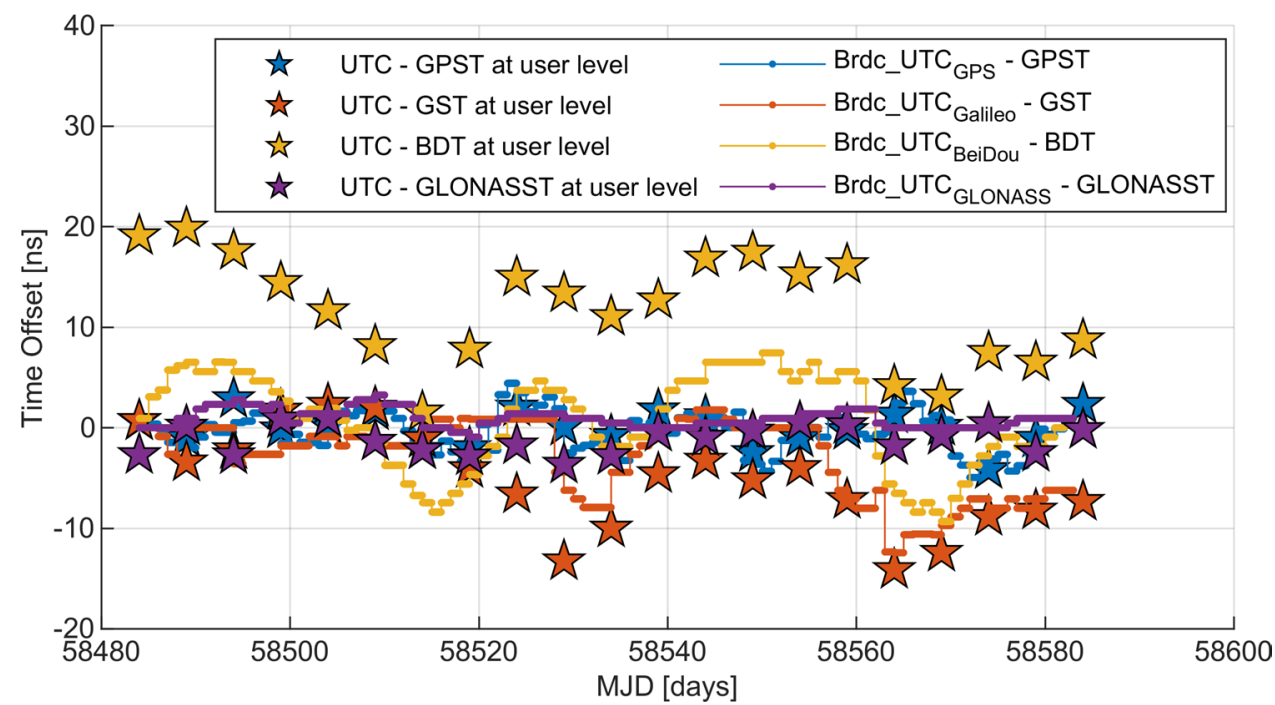




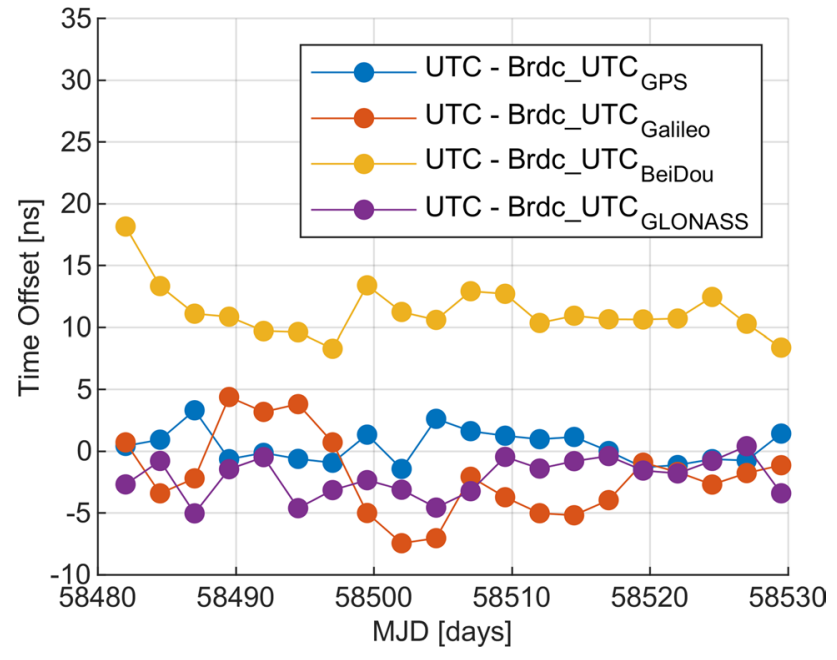

Fig. 14 UTC - Brdc_UTC ${ }_{\mathrm{GNSS}}$, computed via UTC(ORB) in January-April 2019

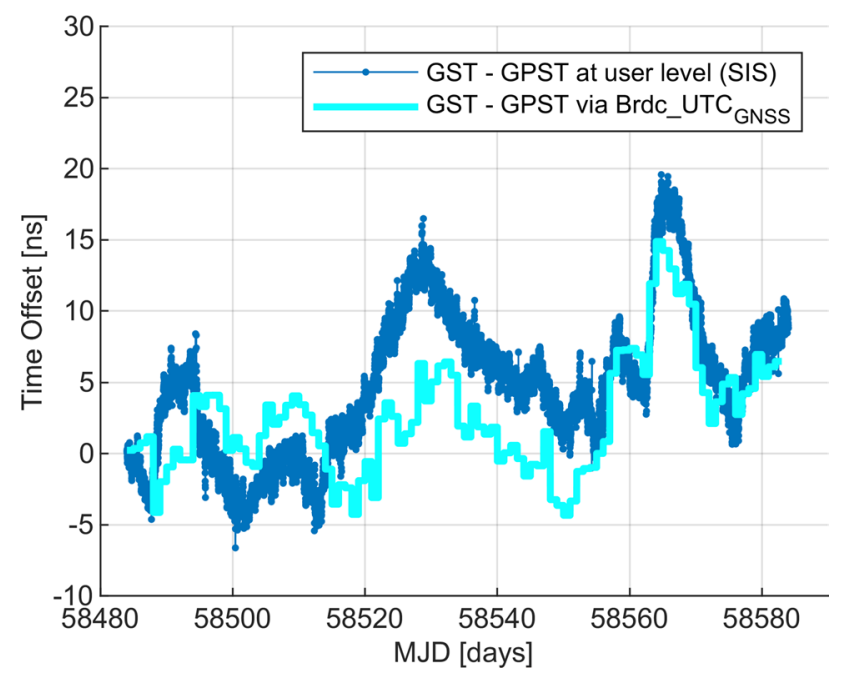

Fig. 15 Comparison of GST - GPST as computed by the different approaches, 2019

obtained via SIS solution and GNSST mean $_{\text {. However, some }}$ bias can be observed when comparing the SIS solution with

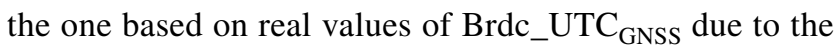
different UTC prediction algorithms and possible calibration residuals of each system, which can be improved in the further development of GNSSs. Overall, the noise of the user GYTO is small (5 ns or less) for both methods. However, it is only representative of high-precision receivers such as those used in the tests; the actual performance would depend greatly on the quality of the user receivers, regardless of which method is preferred at the system level.

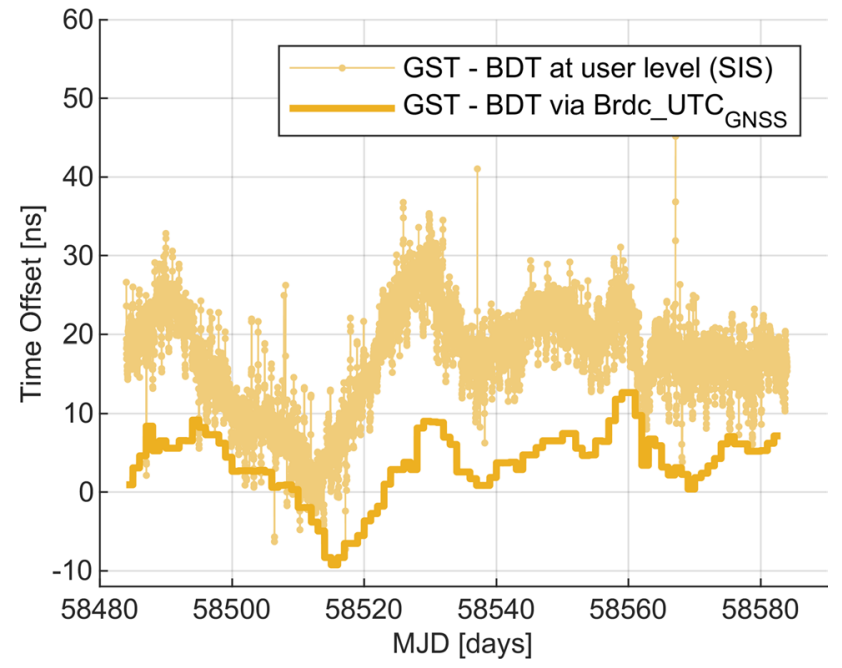

Fig. 16 Comparison of GST - BDT as computed by the different approaches, 2019

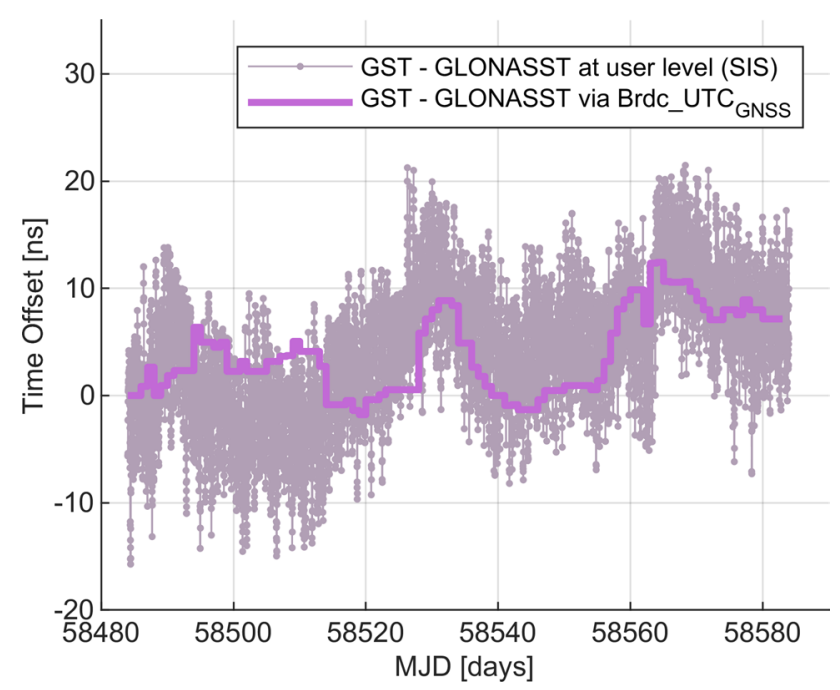

Fig. 17 Comparison of GST - GLONASST as computed by the different approaches, 2019

\section{Discussion and conclusions}

We presented a study concerning GNSS interoperability topics from a timing point of view, i.e., about the intersystem time offsets XYTO. These parameters can be very useful when using two or more constellations at the same time to fix a position. While the XYTO parameters can be estimated by the user in major cases, broadcasting the information can help the user in situations where not enough satellites are visible to determine XYTO, for example, in urban canyons. To avoid the need to broadcast all the intersystem biases, we 
Fig. 18 GYTO differences at the user level between the user SIS solution and the solution based on Brdc_UTC ${ }_{\text {GNSS }}$ in 2019. The mean value and standard deviation of these differences are also reported in the legend

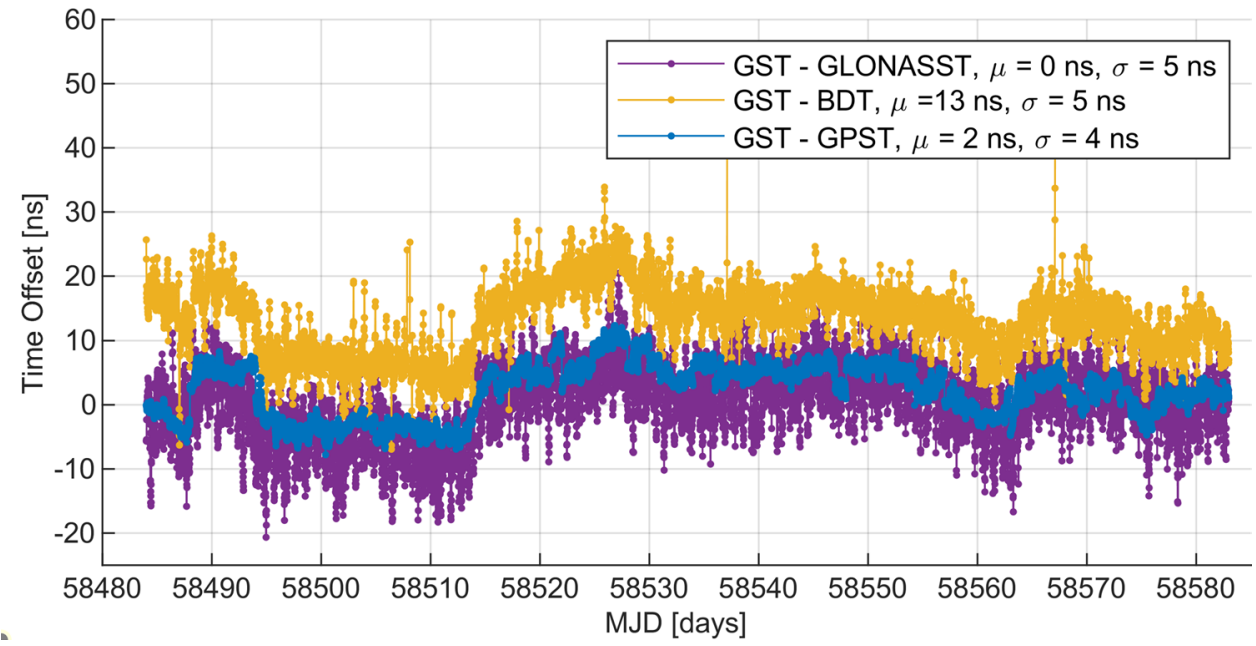

GYTO performance at user level for the period analyzed

Mean value $(\mu)$ Standard

[ns] deviation $(\sigma)$

[ns]

\begin{tabular}{lll}
\hline Differences between SIS solution and GNSST mean $_{\text {-based solution }}$ & & \\
GST - GPST & 0 & 2 \\
GST - BDT & 0 & 2 \\
GST - GLONASST & 0 & 4 \\
Differences between SIS solution and Brdc_UTC ${ }_{G N S S}$-based solution & & 3 \\
GST - GPST & 0 & 5 \\
GST - BDT & 3 & 4 \\
GST - GLONASST & 8 & \\
\hline
\end{tabular}

proposed two different methods of XYTO dissemination, which require either one or no additional parameters in the navigation message of each constellation. The basic idea is to broadcast the difference between the GNSS time and a reference common to all GNSSs in each GNSS navigation message.

In the first approach, this common reference is proposed to be a simple mean (GNSST $_{\text {mean }}$ ) of the reference time scales of the four global GNSSs (BeiDou, Galileo, GLONASS, GPS), implying changes to the GNSS design for the computation of GNSST $_{\text {mean }}$ and its inclusion in the navigation messages. Moreover, the realization of GNSST $_{\text {mean }}$ will also need to be agreed upon by all systems involved to avoid misalignments in the results. Possible issues could come from different implementations, which is why GNSST mean $_{\text {}}$ computation is proposed here to be as simple as possible. All of this contributes, however, to a lower degree of autonomy between the systems. For example, an outage at one GNSS provider would impact the estimation of GNSST $_{\text {mean }}$ and its prediction in each system.

As the realistic presented scenario shows, the results are promising as long as the GNSS stations of the provider and user are properly calibrated. Under nominal conditions, the satellite visibility in different regions has a negligible impact, as shown with the chosen distribution of the stations involved. Our results have shown that for fully calibrated stations, the accuracy of the XYTO determined at the user level from the broadcast GNSST - GNSST mean $_{\text {is less than }}$ 5 ns. Any residual calibration error at the user level or at the system level introduces a corresponding error in XYTO. Considering that calibration residuals of a few nanoseconds are quite common, the overall uncertainty could be at the level of $7-8 \mathrm{~ns}(1 \sigma)$. If the user receiver is not calibrated at all, this component can be even larger when using the broadcast XYTO, but in such poor visibility conditions, there will be a large timing and positioning error in any case.

In our second proposed approach, the common reference relies instead on a parameter already available and broadcast by each GNSS provider: the predicted difference GNSST - UTC. Each system provides a prediction of this offset using a realization of UTC maintained by one or several time laboratories contributing to the UTC computed by the BIPM. This second approach relies on the assumption that such UTC predictions are equivalent. The performance 
of the method is, therefore, linked to the validity of this assumption and hence to the quality of the predictions of GNSST - UTC as broadcast by GNSS systems. Currently, the uncertainty includes the difference between GNSST $_{i}$ - UTC prediction algorithms, the contribution of different real-time UTC realizations used as a reference, and the update rate of the UTC parameter in the navigation message of visible satellites. Based on data from 2018 and 2019, our results indicate that this second approach allows the user to retrieve the intersystem biases with an error smaller than $10 \mathrm{~ns}$, which is similar to or slightly larger than the previous method. We can expect that this will improve with the further improvement of the GNSS systems connecting to the UTC.

In both cases, the users should be able to use the GNSST $_{\text {mean }}$ or UTC information to obtain XYTO. A comparison between the two proposed methods in terms of complexity at various levels and impacts of various factors is reported in Table 3.

This comparison highlighted that the second approach, based on the broadcast UTC parameters, alleviates the

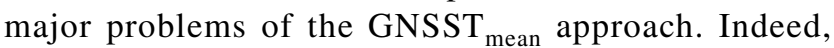
considering that each system is already transmitting its own estimation of GNSST - UTC, there is no need for constructing a new paper time scale and no need for extra parameters in the navigation message or any dedicated infrastructure. Therefore, at the system level, no changes must be implemented. Furthermore, this lack of change implementation allows us to avoid the proliferation of time references. Therefore, the main challenge to GNSS providers is to keep their own reference time as close as possible to the international reference UTC and to improve their own prediction of GNSST - UTC to minimize the errors on XYTO at the user level.

Recent studies have shown that when the user has good satellite visibility, the PVT solution obtained when determining intersystem bias as an additional unknown is similar to or better than when using a broadcast value because this quantity also contains receiver intersystem delays (Defraigne et al 2020). However, in situations of poor visibility, the user would benefit by a priori additional information on the intersystem bias. These situations typically correspond to urban or natural canyons, where the satellite geometry does not make precise positioning possible due to the poor dilution of precision (DOP). In these scenarios, the positioning error is generally at the level of at least tens of meters, and an error of $10 \mathrm{~ns}$ as obtained in the two proposed approaches of the current study will not induce a significant degradation of the PVT solution.

\section{Appendix: Receiver calibration}

A time offset measurement or estimation always implies an accurate calibration of the equipment used. In this Appendix, we present the details of the calibration procedures carried out on the GNSS receiver before using their measurements and pseudoranges for the reported case studies. Among the multi-GNSS stations considered, only BRUX was already calibrated for GPS and Galileo signals, and BRUX was then differentially calibrated for GLONASS using information from BIPM Circular $\mathrm{T}$ and for BeiDou using the information found in BeiDou documentation. Then, a differential calibration with respect to BRUX was carried out for the other stations involved in the case studies.

Table 3 Qualitative comparison of the two proposed methods

\begin{tabular}{|c|c|c|}
\hline Issues & Method 1: GNSST mean $_{\text {based }}$ & Method 2: Brdc_UTC ${ }_{\text {GNSS }}$ based \\
\hline Complexity at provider level & $\begin{array}{l}\text { Very high, as GNSST mean is not yet available } \\
\text { (impact on system design, ICD, etc.). The } \\
\text { capacity to compute, predict and disseminate } \\
\text { GNSST }_{\text {mean }} \text { should be agreed among all GNSSs; } \\
\text { an infrastructure needs to be put in place by } \\
\text { each provider for the generation and valida- } \\
\text { tion of GNSST mean and the navigation message } \\
\text { structure needs to be updated. }\end{array}$ & None, as Brdc_UTC ${ }_{\mathrm{GNSS}}$ are already available. \\
\hline Coordination among system providers & Necessary & Not necessary \\
\hline Impact from one GNSS data outages & $\begin{array}{l}\text { Possible jumps or unavailability of one GNSST } \\
\text { directly impacts the capacity of generating } \\
\text { GNSST }_{\text {mean }} \text { for the other providers. }\end{array}$ & $\begin{array}{l}\text { XYTO performance not changed for the other } \\
\text { systems still operating nominally }\end{array}$ \\
\hline Impact of calibration errors at provider level & $\begin{array}{l}\text { High, as all receivers at system level need to be } \\
\text { fully calibrated for all other GNSS signals }\end{array}$ & $\begin{array}{l}\text { High, as each GNSS provider needs to fully } \\
\text { calibrate its chain to UTC }\end{array}$ \\
\hline Impact of low visibility at user level & \multicolumn{2}{|c|}{ Similar impact, as the user will have to use the broadcast information in any case } \\
\hline Complexity for receiver manufacturers & \multicolumn{2}{|c|}{$\begin{array}{l}\text { Similar complexity level, as they will have to update their software to estimate XYTO from the } \\
\text { proposed broadcast information }\end{array}$} \\
\hline
\end{tabular}




\section{Calibration of the BRUX receiver for GLONASS and BeiDou signals}

The calibration of GLONASS measures is based on BIPM data, where the difference between UTC and GLONASS time is reported. Figure 19 shows GLONASS time versus UTC (ORB) uncalibrated measures per satellite, as obtained through the SIS by the BRUX receiver; red dots are instead the calibrated estimates of the same quantity obtained through BIPM data according to Equation (6).

$$
\begin{aligned}
& {[\mathrm{UTC}(\mathrm{ORB})-\text { GLONASST }]_{\mathrm{BIPM}}} \\
& \quad=[\mathrm{UTC}-\mathrm{GLONASST}]_{\mathrm{BIPM}}-[\mathrm{UTC}-\mathrm{UTC}(\mathrm{ORB})]_{\mathrm{BIPM}}
\end{aligned}
$$

Since GLONASS uses Frequency Division Multiple Access (FDMA), interfrequency biases between the different satellite signals have been determined and applied to BRUX data to obtain a calibrated solution (Fig. 20). The calibration values for each satellite were computed as the mean value for the 2-month test period.

For BeiDou calibration, as BIPM does not yet publish data related to BeiDou, we combined UTCr - BDT values reported by Cai et al. at ICG 2017 with the BIPM's UTCr - UTC(ORB), represented by Equation (7). Figure 21 shows the result of such computation, which relies on a calibration made within the frame of the BeiDou system.

$$
\begin{aligned}
& {[\mathrm{UTC} r-\mathrm{BDT}]_{\mathrm{ICG} 2017}-[\mathrm{UTC} r-\mathrm{UTC}(\mathrm{ORB})]_{\mathrm{BIPM}}} \\
& \quad=[\mathrm{UTC}(\mathrm{ORB})-\mathrm{BDT}]_{\text {Calibrated }}
\end{aligned}
$$

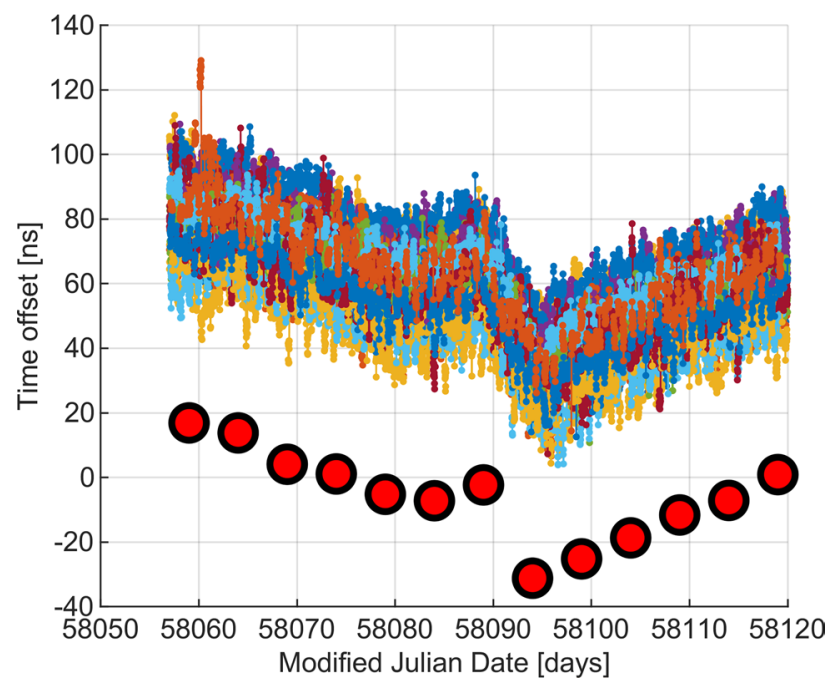

Fig. 19 Uncalibrated UTC(ORB) - GLONASST measurements per satellite from ORB receiver versus BIPM data (red dots)

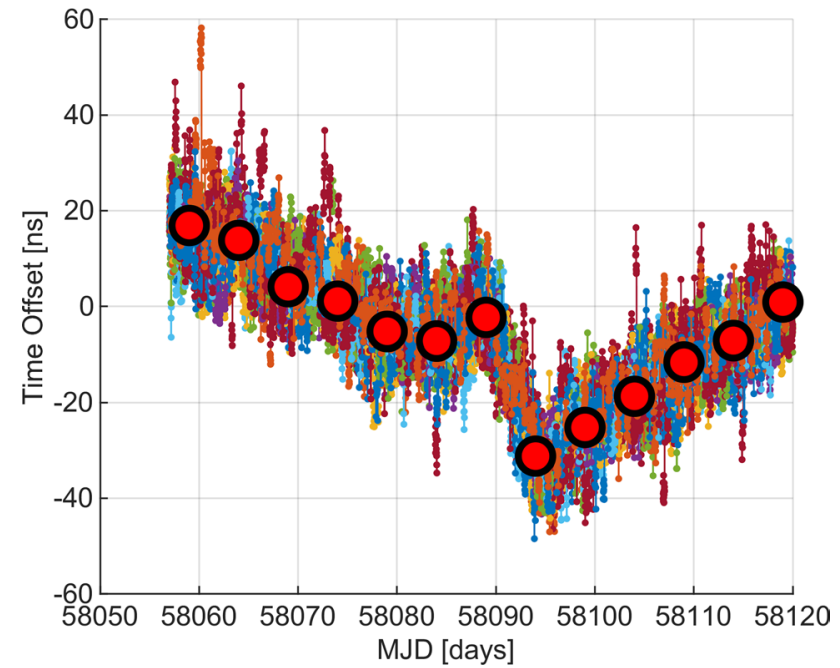

Fig. 20 UTC(ORB) - GLONASST measurements per satellite from the ORB receiver after differential calibration versus BIPM data (red dots)

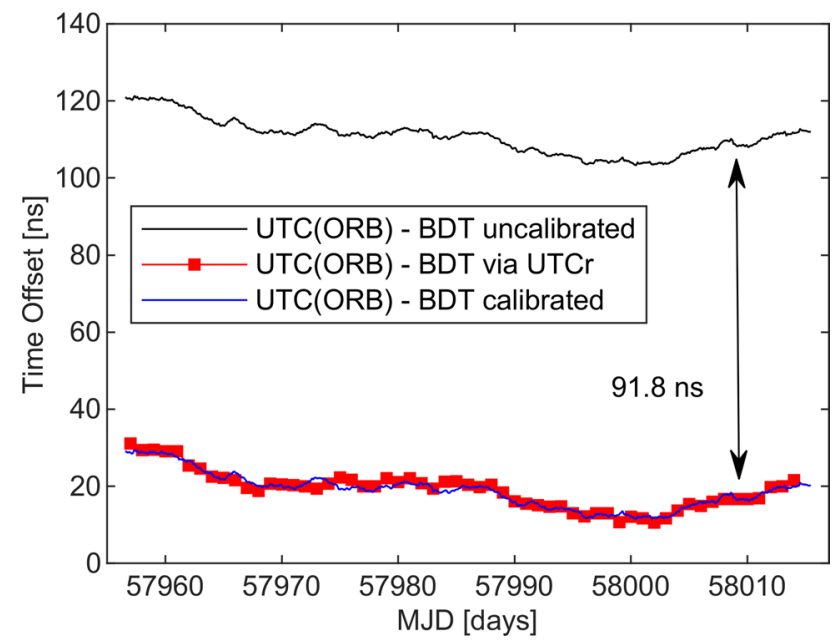

Fig. 21 UTC(ORB) - BDT calibrated and uncalibrated estimations

\section{Differential calibration of other stations versus BRUX}

The other multi-GNSS stations (Table 1) were then calibrated differentially with respect to the fully calibrated BRUX station. We chose the analysis period where there were no maintenance activities ongoing at the five selected stations.

A different approach was needed to determine the calibration corrections for the other stations, as these stations were not calibrated for any of the four GNSS constellations, and there was no other external calibrated quantity that could be used to calibrate them. Here, we consider the intersystem differences between GNSSs via GNSST $_{\text {mean }}$; 
therefore, we calibrated such quantities of the other stations with respect to the same quantities that were fully calibrated for BRUX. For GLONASS, the interfrequency bias of each satellite was also considered using values previously obtained for the BRUX station.

Funding Open Access funding provided by Istituto Nazionale di Ricerca Metrologica.

Open Access This article is licensed under a Creative Commons Attribution 4.0 International License, which permits use, sharing, adaptation, distribution and reproduction in any medium or format, as long as you give appropriate credit to the original author(s) and the source, provide a link to the Creative Commons licence, and indicate if changes were made. The images or other third party material in this article are included in the article's Creative Commons licence, unless indicated otherwise in a credit line to the material. If material is not included in the article's Creative Commons licence and your intended use is not permitted by statutory regulation or exceeds the permitted use, you will need to obtain permission directly from the copyright holder. To view a copy of this licence, visit http://creativecommons.org/licenses/by/4.0/.

\section{References}

Chinese Satellite Navigation Office (2018) BeiDou Navigation Satellite System Signal In Space Interface Control Document; Open Service Signal B3I Version 1.0 (BDS-SIS-ICD-B3I-1.0). Retrieved from http://BeiDou.gov.cn/xt/gfxz/201802/P02018020962360 1401189.pdf

Defraigne P, Petit G (2015) CGGTTS-Version 2E: CGGTTS-version 2E: an extended standard for GNSS time transfer. Metrologia 52(6):G1

Defraigne P, Guyennon N, Bruyninx C (2008) GPS time and frequency transfer: PPP and phase-only analysis. Int J Navigation Observation. https://doi.org/10.1155/2008/175468

Defraigne P, Pinat E, Bertrand B (2020) Impact of Galileo-to-GPStime-offset accuracy on multi-GNSS positioning and timing. GPS Solutions (accepted for publication).

Gioia C, Borio D (2016) A statistical characterization of the Galileoto-GPS inter-system bias. J Geodesy 90(11):1279-1291

GLONASS Coordination Scientific Information Center (1998) Global Navigation Satellite System GLONASS Interface Control Document Version 4.0. Available at: https://www.unavco.org/help/gloss ary/docs/ICD_GLONASS_4.0_(1998)_en.pdf

GSA (2016a) European GNSS (Galileo) Open Service Signal-In-Space Interface Control Document Issue 1 Revision 3 (Galileo OS SIS ICD Issue 1.3) https://www.gsc-europa.eu/system/files/galileo_ documents/Galileo-OS-SIS-ICD.pdf

GSA (2016b) European GNSS (Galileo) Initial Services - Open Service - Service Definition Document, Issue 1 Revision 0 (Galileo OS SDD Issue 1.0), superseded by Issue 1 Revision 1 May 2019 available at https://www.gsc-europa.eu/sites/default/files/sites/all/ files/Galileo-OS-SDD_v1.1.pdf

GSA (2018) European GNSS (Galileo) Initial Services Open Service Quarterly Performance Report January - March 2018. Available at: https://www.gsc-europa.eu/system/files/galileo_documents/ Galileo-IS-OS-Quarterly-Performance Report-Q1-2018.pdf

Kosheliaevskii NB (2019) Thinking on GNSS system time scale and interoperability. In: Proceedings of ION PTTI 2019, Reston, Virginia, USA, January 28-31, pp 117-121

Noll CE (2010) The crustal dynamics data information system: a resource to support scientific analysis using space geodesy. Adv Space Res 45(12): 1421-1440

Panfilo G, Arias F (2019) The Coordinated Universal Time (UTC) Metrologia 56(4):042001

Petit G, Defraigne P, Warrington B, Uhrich P (2006) Calibration of dual frequency GPS receivers for TAI. In: Proceedings of the 20th European Frequency and Time Forum, Braunschweig, pp 455459. Retrived from https://www.eftf.org/fileadmin/conferences/ eftf/documents/Proceedings/proceedingsEFTF2006.pdf

Píriz R, Mozo A, Tobías G, Fernandez V, Tavella P, Sesia I, Cerretto G, Hahn J (2008) GNSS interoperability: offset between reference time scales and timing biases. Metrologia 45(6):87-102

Signorile G, Sesia I, Thai TT, Defraigne P, Tavella P (2018) Galileo and GNSS time offsets. In: Proceedings of the 32nd European time and frequency forum, Turin, pp 276-280, https://doi. org/10.1109/EFTF.2018.8409048

United States Space Force (2013) Interface Specification IS-GPS-200 Revision H. Retrieved from https://www.gps.gov/technical/icwg/ IS-GPS-200H.pdf

Tavella P, Petit G (2019) On the use of UTC to support GNSS interoperability. In: ICG-14 WG-S, Bangalore, India, 2019

Verhasselt K, Defraigne P (2019) Multi-GNSS time transfer based on the CGGTTS. Metrologia 56(6):065003. https://doi. org/10.1088/1681-7575/ab3ed7

Waller P, Valceschini R, Delporte J, Valat D (2019) Cross-calibrations of multi-GNSS Receiver Chains. In: Proceedings of 2019 joint conference of the IEEE international frequency control symposium and European frequency and time forum, Orlando, pp 1-4. https://doi.org/10.1109/FCS.2019.8856134

Yuan H, Guang W, Zhang J (2019) A comparative study on different GNSS time offsets monitoring methods. In: ICG-14 WG-S, Bangalore, India, 2019

Publisher's Note Springer Nature remains neutral with regard to jurisdictional claims in published maps and institutional affiliations.

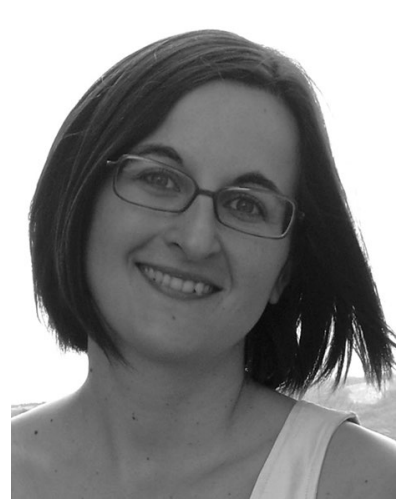

Ilaria Sesia received the M.S. degree in communication engineering and a Ph.D. degree in metrology from Politecnico di Torino in 2003 and 2008. She is a senior researcher at the Italian Metrological Institute (INRiM), where she coordinates the EGNSS metrological timekeeping activities. Since 2004, she has been deeply involved in the design and development of the European navigation system Galileo. 


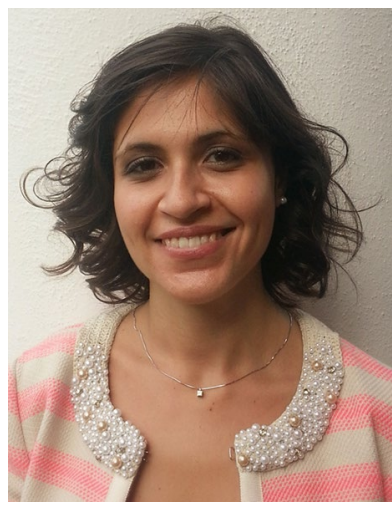

Giovanna Signorile received the M.S. degree in telecommunication engineering from the Politecnico di Torino in 2011. She works at the Italian Metrology Institute (INRIM), Torino, Italy. She is involved in metrological time-keeping activities, mainly focused on E-GNSS timing aspect validation within several ESA and GSA projects and on time scale generation algorithms, also applied to the Italian time scale.

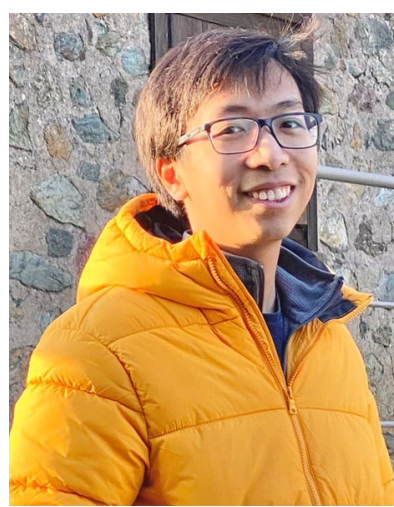

Tung Thanh Thai graduated as an engineer in telecommunications at Hanoi University of Technology, Vietnam, and received an M.Sc. in Navigation and Related Applications at Politecnico di Torino, Italy. He is now a fellow researcher at the Italian Metrology Institute (INRIM) and is involved in various activities regarding GNSS timing systems, two-way satellite time and frequency transfer technique and time scale algorithms.

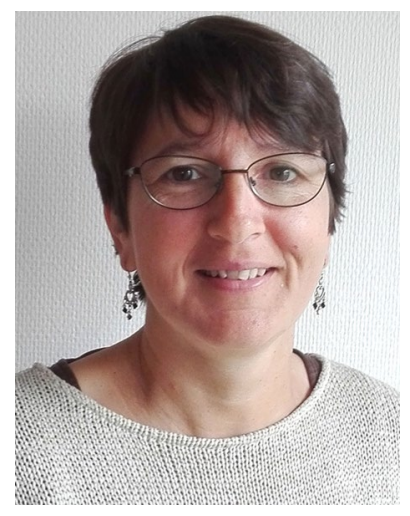

Pascale Defraigne is Project Leader at the Royal Observatory of Belgium for research activities involving GNSS time transfer, time scale generation and ionospheric sounding based on GNSS measurements. Since 2012, Pascale Defraigne has assumed the Chair of the Working Group on GNSS Time Transfer for the Consultative Committee for Time and Frequency, a subcommittee of the International Committee for Weights and Measurements.

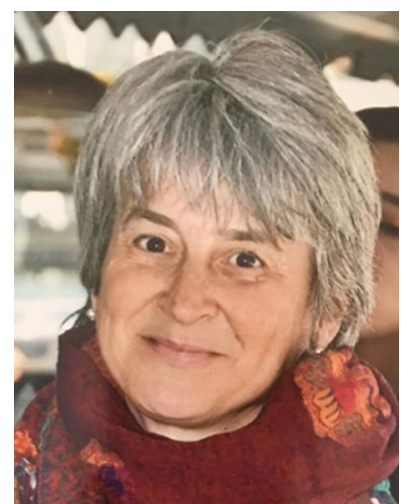

Patrizia Tavella degree in Physics and Ph.D. in Metrology, is the Director of the Time Department of the Bureau International des Poids et Mesures after 30 years at the Italian Metrology Institute, INRIM. Her main interests are mathematical and statistical models applied to atomic time scales. She was deeply involved in the development of the European Navigation System Galileo. 